\title{
Intersecting electric and magnetic $p$-branes: spherically symmetric solutions
}

\author{
K.A. Bronnikov ${ }^{1} \dagger$, U. Kasper ${ }^{2} \ddagger$ and M. Rainer \\ $\dagger$ Centre for Gravitation and Fundamental Metrology \\ VNIIMS, 3-1 M. Ulyanovoy St., Moscow 117313, Russia \\ $\ddagger$ Institut für Mathematik, Universität Potsdam, \\ PF 601553, D-14415 Potsdam, Germany
}

\begin{abstract}
We consider a $D$-dimensional self-gravitating spherically symmetric configuration of a generalized electro-magnetic $n$-form $F$ and a dilatonic scalar field, admitting an interpretation in terms of intersecting $p$-branes. For theories with multiple times, selection rules are obtained, which obstruct the existence of $p$-branes in certain subspaces. General static solutions are obtained under a specific restriction on the model parameters, which corresponds to the known "intersection rules". More special families of solutions (with equal charges for some of the $F$-field components) are found with weakened restrictions on the input parameters. Black-hole solutions are determined, and it is shown that in the extreme limit the Hawking temperature may tend to zero, a finite value, or infinity, depending on the $p$-brane intersection dimension. A kind of no-hair theorem is obtained, claiming that black holes cannot coexist with a quasiscalar component of the $F$-field.
\end{abstract}

\section{Introduction}

This paper studies some possible gravitational effects of multidimensional unification schemes with hypermembranes, currently widely discussed as so-called M-theories (see reviews in [1-5]) and are closely related to earlier supergravity theories [6, 7]. These models contain in their lowenergy bosonic sectors sets of antisymmetric Maxwell-like forms $F$ of various ranks (connected with highly symmetric, usually flat, subspaces of space-times of 10 and more dimensions), interacting with dilatonic scalar fields.

We discuss static, spherically symmetric systems. Trying to adhere to the most realistic conditions, we restrict the consideration to a single $n$-form $F$ (since in 4 dimensions we only deal with a single electromagnetic field), interacting with a single scalar field, and to ordinary $S^{2}$ spheres, although the solution technique is applicable to more general systems.

Nevertheless, we admit the existence of all possible types of components of $F$-fields compatible with spherical symmetry, namely, electric, magnetic and quasiscalar ones. It turns out possible to express the general exact solutions in terms of elementary functions, if the input parameters of the model satisfy certain orthogonality conditions in minisuperspace.

\footnotetext{
${ }^{1} \mathrm{e}-m a i l:$ kb@goga.mainet.msk.su

${ }^{2}$ e-mail: ukasper@rz.uni-potsdam.de

${ }^{3}$ e-mail: mrainer@aip.de
} 
These conditions correspond to the known $p$-brane intersection rules of $M$-theories. For the latter many solutions have been obtained [8-14], which here coincide with some special cases of our solutions below.

It is possible to weaken the restrictions upon the model parameters, and nevertheless to find special families of solutions, which have the additional symmetry, that some $F$-field charges coincide. An example is a solution with equal electric and magnetic charges.

Among our solutions, we also select those describing black holes. It turns out that a black hole cannot coexist with a nonzero quasiscalar component of the $F$-field. This result generalizes the well-known no-hair theorems.

The black hole solutions depend on 3 integration constants, related to the electric, the magnetic, and the mass charge. It is also shown that the Hawking temperature of such black holes depends on the intersection dimension $d_{\text {isc }}$ of the corresponding $p$-branes. In the extreme limit the black hole temperature may tend to zero for $d_{\text {isc }}=0$, a finite limit for $d_{\text {isc }}=1$, and infinity for $d_{\text {isc }}>1$.

Similar sets of solutions with a smaller number of integration constants are obtained for more general models, with an additional symmetry, e.g. equal electric and magnetic charges.

The paper is organized as follows: Sect. 2 describes the general model. Sect. 3 discusses the field equations and defines the minisuperspace representation. Sect. 4 outlines the general construction of solutions using an orthogonality condition (Sect. 4.1), and a simplified method (with less restrictions) for the case of equal charges (Sect. 4.2). In Sect. 5 singularities and conditions of black holes are investigated. Sect. 6 treats more specific electro-magnetic type solutions. We give a general solution for a special model (Sect. 6.1), give examples for this solution (Sect. 6.2) and present a special solution (with equal charges as additional symmetry) for a more general model (Sect. 6.3). Sect. 7 concludes with final remarks on the main results.

For convenience we now list some notional conventions for indices and their corresponding objects used below:

$L, M, P \quad \mapsto$ coordinate labels of the $D$-dimensional Riemannian space M;

$I, J, \ldots \quad \mapsto \quad$ subsets of $I_{0}:=\{0,1, \ldots, N\}$;

e, $\mathrm{m} \quad \mapsto \quad$ labels of electric resp. magnetic type forms;

$s, s^{\prime} \quad \mapsto \quad$ unified indices, eI or $\mathrm{m} I$

$i, j, \ldots \quad \mapsto \quad$ labels of subspaces of $\mathrm{M}$;

$A, B, \ldots \quad \mapsto \quad$ minisuperspace coordinate labels.

As usual, we use the summation convention over repeated indices with one index in lower the other in upper position.

\section{The model}

We consider a $D$-dimensional classical bosonic field theory with the action

$$
S=\int d^{D} x \sqrt{g}\left(R-\varphi^{, M} \varphi, M-\frac{\eta_{F}}{n !} F^{2} \mathrm{e}^{2 \lambda \varphi}\right)
$$

where $g=\left|\operatorname{det} g_{L M}\right|, L, M=0, \ldots, D-1, R$ is the scalar curvature, $\varphi$ is a scalar matter field, and $\lambda$ is a coupling constant; furthermore,

$$
\begin{gathered}
F^{2} \equiv F^{M_{1}, \ldots, M_{n}} F_{M_{1}, \ldots, M_{n}}, \quad n=2,3, \ldots, D-2 \\
F=d U \quad \text { i.e. } \quad F_{M_{1}, \ldots, M_{n}}=\partial_{\left[M_{1}\right.} U_{\left.M_{2}, \ldots, M_{n}\right]},
\end{gathered}
$$


where $U$ is a potential $(n-1)$-form and square brackets denote alternation. The coefficient $\eta_{F}= \pm 1$ will be chosen later to provide a positive energy density of the $F$-field.

The field equations read:

$$
\begin{aligned}
& G_{M}^{P} \equiv R_{M}^{P}-\frac{1}{2} \delta_{M}^{P} R=T_{M}^{P}[\varphi]+T_{M}^{P}[F] \\
& \nabla_{M}\left(\mathrm{e}^{2 \lambda \varphi} F^{M M_{2}, \ldots, M_{n}}\right)=0 \\
& \nabla^{M} \nabla_{M} \varphi=\eta_{F} \lambda F^{2} \mathrm{e}^{2 \lambda \varphi}
\end{aligned}
$$

where the energy-momentum tensors (EMTs) are

$$
\begin{aligned}
& T_{M}^{P}[\varphi]=-\varphi, M \varphi^{, P}+\frac{1}{2} \delta_{M}^{P} \varphi_{, L} \varphi^{, L} \\
& T_{M}^{P}[F]=\frac{\eta_{F}}{n !} \mathrm{e}^{2 \lambda \varphi}\left(-F_{M L_{2}, \ldots, L_{n}} F^{P L_{2}, \ldots, L_{n}}+\frac{1}{2} \delta_{M}^{P} F^{2}\right) .
\end{aligned}
$$

We try to find static, spherically symmetric solutions to the set of equations (2.3) to (2.5). We assume a connected multidimensional space-time structure with

$$
\mathrm{M}=M_{-1} \times M_{0} \times M_{1} \times \cdots \times M_{N}, \quad \operatorname{dim} M_{i}=d_{i}, \quad i=0, \ldots, N,
$$

where $M_{-1} \subset \mathbb{R}$ corresponds to a radial coordinate $u, M_{0}=S^{2}$ is a 2-sphere, $M_{1} \subset \mathbb{R}$ is time, and $M_{i}, i>1$ are internal factor spaces. The metric is assumed correspondingly to be

$$
\begin{aligned}
d s^{2} & =\mathrm{e}^{2 \alpha(u)} d u^{2}+\sum_{i=0}^{N} \mathrm{e}^{2 \beta_{i}(u)} d s_{i}^{2} \\
& \equiv-\mathrm{e}^{2 \gamma(u)} d t^{2}+\mathrm{e}^{2 \alpha(u)} d u^{2}+\mathrm{e}^{2 \beta_{0}(u)} d \Omega^{2}+\sum_{i=2}^{N} \mathrm{e}^{2 \beta_{i}(u)} d s_{i}^{2},
\end{aligned}
$$

where $d s_{0}^{2} \equiv d \Omega^{2}=d \theta+\sin ^{2} \theta d \phi^{2}$ is the line element on $S^{2}, d s_{1}^{2} \equiv-d t^{2}$ with $\beta_{1}=: \gamma$, and $d s_{i}^{2}$, $i>1$, are $u$-independent line elements of internal Ricci-flat spaces of arbitrary dimensions $d_{i}$ and signatures $\varepsilon_{i}$.

All fields must be compatible with spherical symmetry. Hence we assume $\varphi=\varphi(u)$. The $F$-field components may be of electric and magnetic types. An electric-type component is specified by a $u$-dependent potential form

$$
F_{\mathrm{e} I, u L_{2} \ldots L_{n}}=\partial_{[u} U_{\left.L_{2} \ldots L_{n}\right]} \quad U=U_{L_{2}, \ldots, L_{n}} d x^{L_{2}} \wedge \ldots \wedge d x^{L_{n}}
$$

where the coordinate indices $L_{j}$ belong to a certain subspace

$$
M_{I}=M_{i_{1}} \times \cdots \times M_{i_{k}}
$$

of the space-time (2.8), associated with a subset

$$
I=\left\{i_{1}, \ldots, i_{k}\right\} \subset I_{0} \stackrel{\text { def }}{=}\{0,1, \ldots, N\} .
$$

of the set $I_{0}$ of possible factor space numbers. The corresponding dimensions are

$$
d(I) \stackrel{\text { def }}{=} \sum_{i \in I} d_{i}
$$


In the $p$-brane setting [2], one of the coordinates of $M_{I}$ is time, and the form (2.10) describes a $(n-2)$-brane in the remaining subspace of $M_{I}$. By assumption, the subspace $M_{0}$ does not belong to $M_{I}$ (that is, $0 \notin I$ ).

A magnetic-type $F$-form of arbitrary rank $k$ may be defined as a form dual to some electrictype one, namely,

$$
F_{\mathrm{m} I, M_{1} \ldots M_{k}}=\mathrm{e}^{-2 \lambda \varphi}(* F)_{\mathrm{e} I, M_{1} \ldots M_{k}} \equiv \mathrm{e}^{-2 \lambda \varphi} \frac{\sqrt{g}}{k !} \varepsilon_{M_{1} \ldots M_{k} N_{1} \ldots N_{D-k}} F_{\mathrm{e} I}^{N_{1} \ldots N_{D-k}},
$$

where $*$ is the Hodge operator and $\varepsilon$ is the totally antisymmetric Levi-Civita symbol. Thus

$$
\operatorname{rank} F_{\mathrm{m} I}=D-\operatorname{rank} F_{\mathrm{e} I}=d(\bar{I})
$$

where $\bar{I} \stackrel{\text { def }}{=} I_{0} \backslash I$ and nonzero components of $F_{\mathrm{m} I}$ contain indices belonging to the subspace $M_{\bar{I}}$. Since we are considering a single $n$-form, we must put $k=n$ in (2.14), so that

$$
d(I)=n-1 \quad \text { for } \quad F_{\mathrm{e} I}, \quad d(I)=d\left(I_{0}\right)-n=D-n-1 \quad \text { for } \quad F_{\mathrm{m} I} .
$$

As before, the subspace $M_{0}$ does not belong to $M_{I}, 0 \notin I$. So 2.14) describes a magnetic $(D-n-2)$-brane in $M_{I}$.

Let us label all nontrivial components of $F$ by a collective index $s=\left(I_{s}, \chi_{s}\right)$, where $I=$ $I_{s} \subset I_{0}$ characterizes the subspace of $\mathrm{M}$ as described above and $\chi_{s}= \pm 1$ according to the rule

$$
\mathrm{e} \mapsto \chi_{s}=+1, \quad \mathrm{~m} \mapsto \chi_{s}=-1 .
$$

In both the electric and magnetic cases, the set $I$ either does or does not include the number 1 , refering to the external time coordinate. If it does, the corresponding $p$-brane evolves with $t$, and we have a true electric or magnetic field; otherwise the potential (2.10) does not contain any 4-dimensional indices and thus behaves as a scalar in 4 dimensions. In this case we call the corresponding electric-type $F$ component (2.10) "electric quasiscalar" and its dual, magnetictype, $F$ component 2.14) "magnetic quasiscalar". So there are in general four types of $F$-field components:

A. $F_{t u A_{3} \ldots A_{n}}$ - electric $\left(1 \in I, A_{k}\right.$ labeling a coordinate of $\left.M_{l}, l \in I\right)$;

B. $F_{\theta \phi B_{3} \ldots B_{n}}$ - magnetic $\left(1 \in I, B_{k}\right.$ labeling a coordinate of $\left.M_{l}, l \in \bar{I}\right)$;

C. $F_{u A_{2} \ldots A_{n}}$ - electric quasiscalar $\left(1 \notin I, A_{k}\right.$ labeling a coordinate of $\left.M_{l}, l \in I\right)$;

D. $F_{t \theta \phi B_{4} \ldots B_{n}}$ - magnetic quasiscalar $\left(1 \notin I, B_{k}\right.$ labeling a coordinate of $\left.M_{l}, l \in \bar{I}\right)$.

The choice of subspaces $I_{s}$ is arbitrary with the only exception that any two nontrivial components of $F$ must have at least two different indices, otherwise there will appear offdiagonal EMT components, which are forbidden by the Einstein equations, since for our metric the Ricci tensor is diagonal. Evidently, this is a restriction for components of the same (electric or magnetic) type, while any electric component may coexist with any magnetic one. Taking this into account, we may formally consider all $F_{s}$ as independent fields (up to index permutations) each with a single nonzero component.

Let us now pass to the general strategy for solutions, with open number and types of $F$-field components. We denote signatures and logarithms of volume factors of the subspaces of $\mathrm{M}$ as follows:

$$
\prod_{i \in I} \varepsilon_{i}=: \varepsilon(I) ; \quad \sum_{i=0}^{N} d_{i} \beta_{i}=: \sigma_{0}, \quad \sum_{i=1}^{N} d_{i} \beta_{i}=: \sigma_{1}, \quad \sum_{i \in I} d_{i} \beta_{i}=: \sigma(I) .
$$




\section{$3 \quad$ Field equations and minisuperspace}

Let us now exploit the possible dimensional reduction of the present Lagrangian model. The reparametrization gauge on the lower dimensional manifold here is chosen as the (generalized) harmonic one. The variation and the reparametrization gauge of spatially homogeneous cosmological models can be restricted to the time manifold (see e.g. [15, 16]), for spatially inhomogeneous models with homogeneous internal spaces it can be reduced to a lower dimensional (in the cosmological case space-time) manifold (see [17, 18]). In general the dimensional reduction depends on the symmetry of the problem. Here, due to the general spherical symmetry and the Ricci-flat internal spaces, the variation reduces to the radial manifold $M_{-1}$ associated with the radial coordinate, namely $u$. Then, the harmonic gauge makes $u$ a harmonic coordinate, as in $\llbracket 19$, whence $\square u=0$, such that

$$
\alpha(u)=\sigma_{0}(u)
$$

The nonzero Ricci tensor components are then given by

$$
\begin{aligned}
\mathrm{e}^{2 \alpha} R_{t}^{t} & =-\gamma^{\prime \prime}, \\
\mathrm{e}^{2 \alpha} R_{u}^{u} & =-\alpha^{\prime \prime}+\alpha^{\prime 2}-{\gamma^{\prime}}^{2}-2{\beta^{\prime}}^{2}-\sum_{i=2}^{N} d_{i}{\beta_{i}^{\prime}}^{2}, \\
\mathrm{e}^{2 \alpha} R_{\theta}^{\theta} & =\mathrm{e}^{2 \alpha} R_{\phi}^{\phi}=\mathrm{e}^{2 \alpha-2 \beta}-\beta^{\prime \prime}, \\
\mathrm{e}^{2 \alpha} R_{a_{j}}^{b_{i}} & =-\delta_{a_{j}}^{b_{i}} \beta_{i}^{\prime \prime} \quad(i, j=1, \ldots, N),
\end{aligned}
$$

where a prime denotes $d / d u$ and the indices $a_{i}, b_{i}$ belong to the $i$-th internal factor space. The Einstein tensor component $G_{1}^{1}$ does not contain second-order derivatives:

$$
\mathrm{e}^{2 \alpha} G_{1}^{1}=-\mathrm{e}^{2 \alpha-2 \beta}+\frac{1}{2} \alpha^{\prime 2}-\frac{1}{2}\left({\gamma^{\prime}}^{2}+2{\beta^{\prime}}^{2}+\sum_{i=2}^{N} d_{i}{\beta_{i}^{\prime}}^{2}\right) .
$$

The corresponding component of the Einstein equations is an integral of other components, similar to the energy integral in cosmology.

The Maxwell-like equations (2.4) are easily solved and give (with (3.1)):

$$
\begin{aligned}
F_{\mathrm{e} I}^{u M_{2} \ldots M_{n}} & =Q_{\mathrm{e} I} \mathrm{e}^{-2 \alpha-2 \lambda \varphi}, & & Q_{\mathrm{e} I}=\text { const } \\
F_{\mathrm{m} I, u M_{1} \ldots M_{d(\bar{I})}} & =Q_{\mathrm{m} I} \sqrt{\left|g_{\bar{I}}\right|}, & & Q_{\mathrm{m} I}=\mathrm{const}
\end{aligned}
$$

where $\left|g_{\bar{I}}\right|$ is the determinant of the $u$-independent part of the metric of $M_{\bar{I}}$ and $Q_{s}$ are charges. These solutions lead to the following form of the EMTs (2.7) written separately for each $F_{s}$ :

$$
\begin{aligned}
& \mathrm{e}^{2 \alpha} T_{M}^{N}\left[F_{\mathrm{e} I}\right]=-\frac{1}{2} \eta_{F} \varepsilon(I) Q_{\mathrm{e} I}^{2} \mathrm{e}^{2 y_{\mathrm{e} I}} \operatorname{diag}\left(+1,[1]_{I},[-1]_{\bar{I}}\right) \\
& \mathrm{e}^{2 \alpha} T_{M}^{N}\left[F_{\mathrm{m} I}\right]=\frac{1}{2} \eta_{F} \varepsilon(\bar{I}) Q_{\mathrm{m} I}^{2} \mathrm{e}^{2 y_{\mathrm{m} I}} \operatorname{diag}\left(1,[1]_{I},[-1]_{\bar{I}}\right),
\end{aligned}
$$

where the first place on the diagonal belongs to $u$ and the symbol $[f]_{J}$ means that the quantity $f$ takes place on the diagonal for all indices refering to $M_{i}, i \in J$; the functions $y_{s}(u)$ are

$$
y_{s}(u)=\sigma\left(I_{s}\right)-\chi_{s} \lambda \varphi .
$$


The scalar field EMT (2.6) is

$$
\mathrm{e}^{2 \alpha} T_{M}^{N}[\varphi]=\frac{1}{2}\left(\varphi^{a}\right)^{\prime 2} \operatorname{diag}\left(+1,[-1]_{I_{0}}\right) .
$$

The sets $I_{s} \in I_{0}$ may be classified by types A, B, C, D according to the description in the previous section. Denoting $I_{s}$ for the respective types by $I_{A}, I_{B}, I_{C}, I_{D}$, we see from (3.6) that, in order to have positive electric and magnetic energy densities, one has to require

$$
-\varepsilon\left(I_{A}\right)=\varepsilon\left(\bar{I}_{B}\right)=\varepsilon\left(I_{C}\right)=-\varepsilon\left(\bar{I}_{D}\right)=\eta_{F} .
$$

If $t$ is the only time coordinate, (3.9) with $\eta_{F}=1$ holds for any choices of $I_{s}$. If there exist other times, then the relations $(3.9)$ are selection rules for choosing subspaces where the $F$ components may be specified. Especially, they may be of be of importance in unification theories involving multiple times, see 20 .

Here is an example of how the rules (3.9) work. Let there be two time coordinates $x^{0}$ and $x^{4}$ and an electric (A) component of $F$ such that the corresponding subspace $M_{I_{A}}$ does not include the coordinate $x^{4}$ (the electric $p$-brane evolves only with the time $x^{0}$ ). We will express this, by convention, as $I_{A} \ni x^{0}, I_{A} \not \supset x^{4}$. Then for a magnetic (B) component the rules (3.9) imply that $\bar{I}_{B} \not \supset x^{4}$ and consequently $I_{B} \ni x^{4}$. Thus a magnetic $p$-brane must evolve with both times. In a similar way, for $\mathrm{C}$ and $\mathrm{D}$ components of the same $F$-field one easily finds: $I_{C} \not \supset x^{4}, I_{D} \ni x^{4}$.

Returning to the equations, one can notice that each constituent of the total EMT on the r.h.s. of the Einstein equations (2.3) has the property

$$
T_{u}^{u}+T_{\theta}^{\theta}=0 .
$$

As a result, the corresponding combination of Eqs. (2.3) has a Liouville form and is easily integrated:

$$
\begin{aligned}
G_{u}^{u}+G_{\theta}^{\theta} & =\mathrm{e}^{-2 \alpha}\left[-\alpha^{\prime \prime}+\beta_{0}^{\prime \prime}+\mathrm{e}^{2 \alpha-2 \beta_{0}}\right]=0, \\
\mathrm{e}^{\beta_{0}-\alpha} & =s(k, u),
\end{aligned}
$$

where $k$ is an integration constant (IC) and the function $s(.,$.$) is defined as follows:$

$$
s(k, u) \stackrel{\text { def }}{=} \begin{cases}k^{-1} \sinh k t, & k>0 \\ t, & k=0 \\ k^{-1} \sin k t, & k<0\end{cases}
$$

Another IC is suppressed by adjusting the origin of the $u$ coordinate.

With (3.11) the $D$-dimensional line element may be written in the form

$$
d s^{2}=\frac{\mathrm{e}^{-2 \sigma_{1}}}{s^{2}(k, u)}\left[\frac{d u^{2}}{s^{2}(k, u)}+d \Omega^{2}\right]+\sum_{i=1}^{N} \mathrm{e}^{2 \beta_{i}} d s_{i}^{2}
$$

where $\sigma_{1}$ has been defined in (2.18).

We now represent the remaining field equations in midisuperspace, i.e. in $\sigma$-model form 17, 18. Since our reduced manifold $M_{-1}$ is 1-dimensional, here the geometric midisuperspace is in fact just the minisuperspace spanned by the $u$-dependent dilatonic scalar fields. Similar like in 15, 16, we extend this minisuperspace by the matter field, thus treating the whole set of 
unknowns $\beta_{i}(u), \varphi(u)$ as a real-valued vector function $x^{A}(u)$ in an $(N+1)$-dimensional vector space $V$, so that $x^{A}=\beta_{A}$ for $A=1, \ldots, N$ and $x^{N+1}=\varphi$. One can then verify that the field equations for $\beta_{i}$ and $\varphi$ coincide with the equations of motion corresponding to the Lagrangian of a Euclidean Toda-like system

$$
L=\bar{G}_{A B} x^{\prime A} x^{\prime B}-V_{Q}(y), \quad V_{Q}(y)=\sum_{s} \theta_{s} Q_{s}^{2} \mathrm{e}^{2 y_{s}},
$$

where $\theta_{s}$ equals 1 if $F_{s}$ is a true electric or magnetic field and otherwise, if $F_{s}$ is quasiscalar, $\theta_{s}$ equals -1 , according to (3.9). The nondegenerate, symmetric matrix

$$
\left(\bar{G}_{A B}\right)=\left(\begin{array}{cc}
G_{i j} & 0 \\
0 & 1
\end{array}\right), \quad G_{i j}=d_{i} d_{j}+d_{i} \delta_{i j}
$$

defines a positive-definite metric in $V$. The energy constraint corresponding to (3.14) is

$$
E=\sigma_{1}^{\prime 2}+\sum_{i=1}^{N} d_{i}{\beta^{\prime}}_{i}^{2}+\varphi^{\prime 2}+V_{Q}(y)=\bar{G}_{A B} x^{\prime A} x^{\prime B}+V_{Q}(y)=2 k^{2} \operatorname{sign} k,
$$

whith $k$ from (3.11). The integral (3.16) follows here from the $\left(\begin{array}{l}u \\ u\end{array}\right)$ component of (2.3).

The functions $y_{s}(u)$ (3.7) can be represented as scalar products in $V$ (recall that $s=\left(I_{s}, \chi_{s}\right)$ ):

$$
y_{s}(u)=Y_{s, A} x^{A}, \quad\left(Y_{s, A}\right)=\left(d_{i} \delta_{i I_{s}}, \quad-\chi_{s} \lambda\right)
$$

where $\delta_{i I} \stackrel{\text { def }}{=} \sum_{j \in I} \delta_{i j}$ is an indicator for $i$ belonging to $I$ (1 if $i \in I$ and 0 otherwise).

The contravariant components of $Y_{s}$ are found using the matrix $\bar{G}^{A B}$ inverse to $\bar{G}_{A B}$ :

$$
\begin{aligned}
& \left(\bar{G}^{A B}\right)=\left(\begin{array}{cc}
G^{i j} & 0 \\
0 & 1
\end{array}\right), \quad G^{i j}=\frac{\delta^{i j}}{d_{i}}-\frac{1}{D-2} \\
& \left(Y_{s}^{A}\right)=\left(\delta_{i I_{s}}-\frac{d\left(I_{s}\right)}{D-2},-\chi_{s} \lambda\right),
\end{aligned}
$$

and the scalar products of different $Y_{s}$, whose values are of primary importance for the integrability of our system, are

$$
Y_{s, A} Y_{s^{\prime}}^{A}=d\left(I_{s} \cap I_{s^{\prime}}\right)-\frac{d\left(I_{s}\right) d\left(I_{s^{\prime}}\right)}{D-2}+\chi_{s} \chi_{s^{\prime}} \lambda^{2}
$$

\section{Solutions}

\subsection{Orthogonality}

The following assumption makes it possible to entirely integrate the field equations:

The vectors $Y_{s}$ are mutually orthogonal with respect to the metric $\bar{G}_{A B}$, that is,

$$
Y_{s, A} Y_{s^{\prime}}{ }^{A}=\delta_{s s^{\prime}} N_{s}^{2}
$$

(This evidently means that the number of functions $y_{s}$ does not exceed the number of equations.) Due to (2.16), the norms $N_{s}$ are actually $s$-independent:

$$
N_{s}^{2}=d\left(I_{s}\right)\left[1-\frac{d\left(I_{s}\right)}{D-2}\right]+\lambda^{2}=\frac{(n-1)(D-n-1)}{D-2}+\lambda^{2} \stackrel{\text { def }}{=} \frac{1}{\nu},
$$


$\nu>0$. The orthogonality condition (4.1) with (3.20) is a special case of a more general integrability condition found in search for intersecting $p$-brane solutions of Majumdar-Papapetrou type [11].

Due to (4.1), the functions $y_{s}(u)$ obey the decoupled equations

$$
y_{s}^{\prime \prime}=\theta_{s} \frac{Q_{s}^{2}}{\nu} \mathrm{e}^{2 y_{s}},
$$

whence

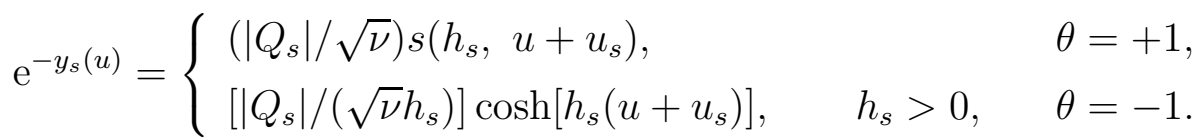

where $h_{s}$ and $u_{s}$ are ICs and the function $s(.,$.$) was defined in (3.12). For the sought functions$ $x^{A}(u)$ we then obtain:

$$
x^{A}(u)=\nu \sum_{s} Y_{s}^{A} y_{s}(u)+c^{A} u+\bar{c}^{A},
$$

where the vectors of ICs $c^{A}$ and $\bar{c}^{A}$ satisfy the orthogonality relations $c^{A} Y_{s, A}=\bar{c}^{A} Y_{s, A}=0$, or

$$
c^{i} d_{i} \delta_{i I_{s}}-\lambda c^{N+1} \chi_{s}=0, \quad \quad \bar{c}^{i} d_{i} \delta_{i I_{s}}-\lambda \bar{c}^{N+1} \chi_{s}=0 .
$$

Specifically, the logarithms of the scale factors $\beta_{i}$ and the scalar field $\varphi$ are

$$
\begin{aligned}
\beta_{i}(u) & =\nu \sum_{s}\left[\delta_{i I_{s}}-\frac{d\left(I_{s}\right)}{D-2}\right] y_{s}(u)+c^{i} u+\bar{c}^{i}, \\
\varphi(u) & =-\lambda \nu \sum_{s} y_{s}(u)+c^{N+1} u+\bar{c}^{N+1},
\end{aligned}
$$

and the function $\sigma_{1}$ which appears in the metric (3.13) is

$$
\sigma_{1}=-\frac{\nu}{D-2} \sum_{s} d\left(I_{s}\right) y_{s}(u)+c^{0} u+\bar{c}^{0}
$$

with

$$
c^{0}=\sum_{i=1}^{N} d_{i} c^{i}, \quad \bar{c}^{0}=\sum_{i=1}^{N} d_{i} \bar{c}^{i} .
$$

Finally, the "conserved energy" $E$ in (3.16) is

$$
E=\nu \sum_{s} h_{s}^{2} \operatorname{sign} h_{s}+\bar{G}_{A B} c^{A} c^{B}=2 k^{2} \operatorname{sign} k .
$$

The relations (3.1), (3.4), (3.5), (3.11), (3.13), (4.4)-(4.11), along with the definitions (3.12) and (4.2) and the restriction (4.1), entirely determine our solution, which is general under the above assumptions.

\subsection{Coinciding charges}

A possible way of integrating the field equations, allowing one to avoid, at least partially, the orthogonality requirement (4.1), is the assumption that some of the functions $y_{s}$ coincide. Indeed, suppose that two functions (3.7), say, $y_{1}$ and $y_{2}$, coincide up to a constant addition (which may be then absorbed by re-defining a charge $Q_{1}$ or $Q_{2}$ ), but the corresponding vectors 
$Y_{1}$ and $Y_{2}$ are neither coinciding, nor orthogonal (otherwise we would have the previously considered situation). Substituting $y_{1} \equiv y_{2}$ into (3.17), one obtains

$$
\left(Y_{1, A}-Y_{2, A}\right) x^{A}=0 .
$$

As all $Y_{s}$ are constants, this is a constraint reducing the number of independent unknowns $x^{A}$. Furthermore, substituting (4.12) to the Lagrange equations for $x^{A}$, one easily finds:

$$
-\left(Y_{1, A}-Y_{2, A}\right) x^{\prime \prime A}=\sum_{s} \theta_{s} Q_{s}^{2} \mathrm{e}^{2 y_{s}} Y_{s}^{A}\left(Y_{1, A}-Y_{2, A}\right)=0 .
$$

In this sum all coefficients of different functions $\mathrm{e}^{2 y_{s}}$ must be zero. Therefore we obtain, first, the orthogonality conditions

$$
Y_{s}^{A}\left(Y_{1, A}-Y_{2, A}\right)=0, \quad s \neq 1,2
$$

for the difference $Y_{1}-Y_{2}$ and other $Y_{s}$, and, second, the following relation for the charges $Q_{1,2}$ :

$$
\left(\nu^{-1}-Y_{1}^{A} Y_{2, A}\right)\left(\theta_{1} Q_{1}^{2}-\theta_{2} Q_{2}^{2}\right)=0,
$$

where Eq. (4.2) is taken into account. The first multiplier in (4.15) is positive $\left(\bar{G}_{A B}\right.$ is positivedefinite, hence a scalar product of two different vectors with equal norms is smaller than their norm squared). Therefore

$$
\theta_{1}=\theta_{2}, \quad Q_{1}^{2}=Q_{2}^{2} .
$$

Imposing the constraints (4.12), (4.14), 4.16), which reduce the numbers of unknowns and integration constants, one simultaneously reduces the number of restrictions on the input parameters (by the orthogonality conditions (4.1)). In other words, a special solution to the field equations may be obtained with a more general initial model. Due to (4.16), this is only possible when the two components with coinciding charges are of equal nature: both must be either true electric/magnetic ones $\left(\theta_{s}=1\right)$, or quasiscalar ones $\left(\theta_{s}=-1\right)$. The solution process may continue as described in the previous subsection, so that the form of the solutions is also similar, but with a reduced number of variables. An explicit example is given below.

\section{$5 \quad$ Singularities and black holes}

Our solutions generalize the well-known spherically symmetric solutions of Einstein and dilaton gravity (see e.g. 21]) and, like these, combine hyperbolic, trigonometric and power functions, depending on the signs of the ICs $k$ and $h_{s}$, so that a considerable diversity of behaviours is possible. It may be asserted, however, that a generic solution possesses a naked singularity at the configuration centre, where $r(u)=\mathrm{e}^{\beta_{0}} \rightarrow 0$. Indeed, without loss of generality, the range of $u$ is $0<u<u_{\max }$, where $u=0$ corresponds to flat spatial infinity, while $u_{\max }$ is finite iff at least one of the constants $h_{s}$ is negative, otherwise and $u_{\max }$ is infinite (by (4.11), $k<0$ is only possible if some $h_{s}<0$ ). In the former case, $u_{\max }$ is the smallest zero in the set of functions

$$
\mathrm{e}^{-y_{s}} \sim \sin \left[\left|h_{s}\right|\left(u-u_{s}\right)\right]
$$

whence it is clear from (4.7) that, at least for some of $i \in\{1, \ldots, n\}$, $\mathrm{e}^{\beta_{i}} \rightarrow \infty$ for $u \rightarrow u_{\max }$. On the other hand, according to (3.13), $\sigma_{1} \rightarrow \infty$, and the coordinate radius shrinks,

$$
r=\mathrm{e}^{\beta_{0}}=\mathrm{e}^{-\sigma_{1}} / s(k, u) \rightarrow 0,
$$


provided the denominator is finite. Hence the limit $u \rightarrow u_{\max }$ is the centre. Such singularities are similar to the Reissner-Nordström repulsive centre, with $g_{t t} \rightarrow \infty$ (if $y_{s}$ in (5.1) corresponds to $\theta_{s}=1$; otherwise some other $\beta_{i}$ becomes infinite) and diverging energy of the respective $F$-field component. Possible coincidences of zeros for different $\mathrm{e}^{-y_{s}}$ do not essentially alter the situation.

Another generic case is that of $u_{\max }=\infty$, when all $h_{s} \geq 0$. Then, as $u \rightarrow \infty$, the factors $\mathrm{e}^{\beta_{i}}$ behave generically like $\mathrm{e}^{k_{i} u}$, with constants $k_{i}$ of either sign, in general different for different $i$. Therefore again we have in most cases a naked singularity, but this time it is not necessarily at the centre. It turns out, however, that this subclass of solutions can describe black holes. So, let us consider the solutions of Subsec. 4.1 and suppose that all $h_{s}>0$ (and hence $k>0$ ) when all asymptotics are exponential, and try to select black hole (BH) solutions. (It can be shown that in the case of only some $h_{s}=0$ there is no $\mathrm{BH}$ solution. A case of interest, when all $h_{s}=0$, may be obtained as a limiting one from the subsequent consideration.)

For BHs we require that all $\left|\beta_{i}\right|<\infty, i=2, \ldots, N$ (regularity of extra dimensions), $|\varphi|<\infty$ (regularity of the scalar field) and $\left|\beta_{0}\right|<\infty$ (finiteness of the spherical radius) as $u \rightarrow \infty$. With $y_{s}(u) \sim-h_{s} u$, this leads to the following constraints on the ICs:

$$
c^{A}=-k \sum_{s}\left(\delta_{1 I_{s}}+\nu Y_{s}^{A} h_{s}\right)
$$

where $A=1$ corresponds to $i=1$. Then, applying the orthonormality relations (4.6) for $c^{A}$, we obtain:

$$
\begin{aligned}
& h_{s}=k \delta_{1 I_{s}}, \\
& c^{A}=-k \delta_{1}^{A}+k \nu \sum_{s} \delta_{1 I_{s}} Y_{s}^{A} .
\end{aligned}
$$

Surprisingly, the "energy condition" (4.11) then holds automatically.

From (5.4) it is obvious that, if at least one $I_{s}$ does not include time $(i=1)$, then $h_{s}=0$, in contrast to our assumption. Actually $h_{s}=0$ means that the corresponding $y_{s}$ has powerlaw asymptotics, uncompensated by exponential asymptotics of other functions. Therefore we conclude: Quasiscalar components of the F-field are incompatible with black holes. This is a kind of no-hair theorem for the case of $p$-branes. We have obtained it for the special case (4.1) when the system is integrable, although very probably it can be proved that the same incompatibility exists for any values of the input parameters. Such a theorem has been proved in 21] for $D$-dimensional dilaton gravity with any value of $\lambda$, while the system is integrable only if $\lambda^{2}=1 /(D-2)$. On the other hand, one can verify that under the conditions (5.4), (5.5) and the additional assumption $\delta_{1 I_{s}}=1$ (that is, only true electric and magnetic fields are present), our solutions indeed describe BHs with a horizon at $u=\infty$. In particular, $g_{t t} \rightarrow 0$ as $u \rightarrow \infty$ and the light travel time $t=\int \mathrm{e}^{\alpha-\gamma} d u$ diverges as $u \rightarrow \infty$. This family exhausts all $\mathrm{BH}$ solutions under the assumptions made, except maybe the limiting case $k=0$.

In what follows, we restrict ourselves to a field with one true electric and one true magnetic components and briefly describe the BH solutions.

\section{Purely electro-magnetic solutions}

Suppose that there are two $F$-field components, Type A and Type B according to the classification of Sec. 2. They will be labelled as $F_{\mathrm{e}}$ and $F_{\mathrm{m}}$ and the corresponding sets $I_{s} \subset I_{0}$ as $I_{\mathrm{e}}$ 
and $I_{\mathrm{m}}$. Then a minimal configuration (2.8) of the space-time M compatible with an arbitrary choice of $I_{s}$ has the following form:

$$
N=5, \quad I_{0}=\{0,1,2,3,4,5\}, \quad I_{e}=\{1,2,3\}, \quad I_{m}=\{1,2,4\},
$$

so that

$$
\begin{array}{ll}
\left(I_{0}\right)=D-1, & d\left(I_{\mathrm{e}}\right)=n-1, \quad d\left(I_{\mathrm{m}}\right)=D-n-1, \quad d\left(I_{\mathrm{e}} \cap I_{\mathrm{m}}\right)=1+d_{2} ; \\
d_{1}=1, & d_{2}+d_{3}=d_{3}+d_{5}=n-2 .
\end{array}
$$

The relations (6.2) show that, given $D$ and $d_{2}$, all $d_{i}$ are known.

In the "polybrane" interpretation [4-7] there is an electric $(n-2)$-brane located on the subspace $M_{2} \times M_{3}$ and a magnetic $(D-n-2)$-brane on the subspace $M_{2} \times M_{4}$. Their intersection dimension $d_{\text {isc }}=d_{2}$ turns out to be of outmost importance for the properties of the solutions.

The index $s$ now takes the two values e and $m$ and

$$
\begin{aligned}
Y_{\mathrm{e}, A} & =\left(1, d_{2}, d_{3}, 0,0,-\lambda\right) ; \quad Y_{\mathrm{m}, A}=\left(1, d_{2}, 0, d_{4}, 0, \lambda\right) ; \\
Y_{\mathrm{e}}^{A} & =(1,1,1,0,0,-\lambda)-\frac{n-1}{D-2}(1,1,1,1,1,0) ; \\
Y_{\mathrm{m}}^{A} & =(1,1,0,1,0, \lambda)-\frac{D-n-1}{D-2}(1,1,1,1,1,0),
\end{aligned}
$$

where the last component of each vector refers to $x^{N+1}=x^{6}=\varphi$.

In the solutions presented below the set of ICs will be reduced by the condition that the space-time be asymptotically flat at spatial infinity $(u=0)$ and by a choice of scales in the relevant directions. Namely, we put

$$
\beta_{i}(0)=\varphi(0)=0 \quad(i=1,2,3,4,5) .
$$

The requirement $\varphi(0)=0$ is convenient and may be always satisfied by re-defining the charges. The conditions $\beta_{i}(0)=0(i>1)$ mean that the real scales of the extra dimensions are hidden in the internal metrics $d s_{i}^{2}$ independent of whether or not they are assumed to be compact.

\subsection{General solution for a special model}

The orthogonality condition (4.1) in our case reads:

$$
\lambda^{2}=d_{2}+1-\frac{1}{D-2}(n-1)(D-n-1)
$$

Being a relation between the input parameters, this restricts the choice of the model; but when the model is chosen in this way, the above solution is general for it.

The solution is entirely determined by the formulae from Subsec. 4.1, where the quantities (6.3) should be put into (4.5) with $\bar{c}^{A}=0$ due to (6.4):

$$
x^{A}(u)=\nu \sum_{s} Y_{s}^{A} y_{s}(u)+c^{A} u ; \quad \mathrm{e}^{-y_{s}(u)}=\left(\left|Q_{s}\right| / \sqrt{\nu}\right) s\left(h_{s}, u+u_{s}\right) .
$$


Due to (6.5) the parameter $\nu$ is

$$
\nu=1 / \sqrt{1+d_{2}} .
$$

The constants are connected by the relations

$$
\begin{array}{ll}
\left(\left|Q_{\mathrm{e}, \mathrm{m}}\right| / \nu\right) s\left(h_{\mathrm{e}, \mathrm{m}}, u_{\mathrm{e}, \mathrm{m}}\right)=1 ; & \\
c^{1}+d_{2} c^{2}+d_{3} c^{3}-\lambda c^{6}=0 ; & c^{1}+d_{2} c^{2}+d_{4} c^{4}+\lambda c^{6}=0 ; \\
\frac{h_{\mathrm{e}}^{2} \operatorname{sign} h_{\mathrm{e}}+h_{\mathrm{m}}^{2} \operatorname{sign} h_{\mathrm{m}}}{1+d_{2}}+G_{i j} c^{i} c^{j}+\left(c^{6}\right)^{2}=2 k^{2} \operatorname{sign} k, &
\end{array}
$$

where the matrix $G_{i j}$ is given in (3.15) and all $\bar{c}^{A}=0$ due to the boundary conditions (6.4). The fields $\varphi$ and $F$ are given by Eqs. (3.4), (3.5), (4.8).

This solution contains 8 independent ICs, namely, $Q_{\mathrm{e}}, Q_{\mathrm{m}}, h_{\mathrm{e}}, h_{\mathrm{m}}$ and 4 others from the set $c^{A}$ constrained by (6.8). All of them are nontrivial constants, unlike those which may be absorbed by a rescaling (shifting $\beta_{i} \rightarrow \beta_{i}+$ const) or a redefinition of the origin of $u$ ( $u \rightarrow u+$ const). It is a direct generalization of the solution for $D=2 n, \lambda=0$ obtained in 114 (the so-called "non-dual" solution for a conformally invariant generalized Maxwell field), the one for $n=2$ ( $D$-dimensional dilaton gravity) and other previous ones (see [21] and references therein). In particular, in dilaton gravity $n=2, d_{2}=0$ and the integrability condition (6.5) just reads $\lambda^{2}=1 /(D-2)$, which is a well-known relation of string gravity. This family, however, does not include the familiar Reissner-Nordström solution, for which $D=4, n=2, \lambda=0, d_{2}=0$ and Eq. (6.5) does not hold.

In the $\mathrm{BH}$ case ((5.4), (5.5) with $\left.\delta_{1 I_{s}}=1\right)$ the solution is more transparent after a coordinate transformation $u \mapsto R$, given by the relation

$$
\mathrm{e}^{-2 k u}=1-2 k / R,
$$

which leads to

$$
\begin{aligned}
& d s^{2}=-\frac{1-2 k / R}{P_{\mathrm{e}}^{B} P_{\mathrm{m}}^{C}} d t^{2}+P_{\mathrm{e}}^{C} P_{m}^{B}\left(\frac{d R^{2}}{1-2 k / R}+R^{2} d \Omega^{2}\right)+\sum_{i=2}^{5} \mathrm{e}^{2 \beta_{i}(u)} d s_{i}^{2}, \\
& \mathrm{e}^{2 \beta_{2}}=P_{\mathrm{e}}^{-B} P_{\mathrm{m}}^{-C}, \quad \mathrm{e}^{2 \beta_{3}}=\left(P_{\mathrm{m}} / P_{\mathrm{e}}\right)^{B}, \\
& \mathrm{e}^{2 \beta_{4}}=\left(P_{\mathrm{e}} / P_{\mathrm{m}}\right)^{C}, \quad \mathrm{e}^{2 \beta_{5}}=P_{\mathrm{e}}^{C} P_{\mathrm{m}}^{B}, \\
& e^{2 \lambda \varphi}=\left(P_{\mathrm{e}} / P_{\mathrm{m}}\right)^{2 \lambda^{2} /\left(1+d_{2}\right)}, \\
& F_{01 M_{3} \ldots M_{n}}=-Q_{\mathrm{e}} /\left(R^{2} P_{\mathrm{e}}\right), \quad F_{23 M_{3} \ldots M_{n}}=Q_{\mathrm{m}} \sin \theta
\end{aligned}
$$

with the notations

$$
\begin{aligned}
& P_{\mathrm{e}, \mathrm{m}}=1+p_{\mathrm{e}, \mathrm{m}} / R, \quad p_{\mathrm{e}, \mathrm{m}}=\sqrt{k^{2}+\left(1+d_{2}\right) Q_{\mathrm{e}, \mathrm{m}}^{2}}-k ; \\
& B=\frac{2(D-n-1)}{(D-2)\left(1+d_{2}\right)}, \quad C=\frac{2(n-1)}{(D-2)\left(1+d_{2}\right)} .
\end{aligned}
$$

The $\mathrm{BH}$ gravitational mass as determined from a comparison of (6.10) with the Schwarzschild metric for $R \rightarrow \infty$ is

$$
G_{N} M=k+\frac{1}{2}\left(B p_{\mathrm{e}}+C p_{\mathrm{m}}\right),
$$


where $G_{N}$ is the Newtonian gravitational constant. This expression, due to $k>0$, provides a restriction upon the charge combination for a given mass, namely,

$$
B\left|Q_{\mathrm{e}}\right|+C\left|Q_{\mathrm{m}}\right|<2 G_{N} M / \sqrt{1+d_{2}}
$$

The inequality is replaced by equality in the extreme limit $k=0$. For $k=0$ our BH turns into a naked singularity (at the centre $R=0$ ) for any $d_{2}>0$, while for $d_{2}=0$ the zero value of $R$ is not a centre $\left(g_{22} \neq 0\right)$ but a horizon. In the latter case, if $\left|Q_{e}\right|$ and $\left|Q_{m}\right|$ are different, the remaining extra-dimensional scale factors are smooth functions for all $R \geq 0$.

The Hawking temperature $T$ of a static, spherical BH can be found, according to [22], from the relation

$$
k_{\mathrm{B}} T=\kappa / 2 \pi, \quad \kappa=\left(\sqrt{\left|g_{00}\right|}\right)^{\prime} /\left.\sqrt{g_{11}}\right|_{\text {horizon }}=\left.\mathrm{e}^{\gamma-\alpha}\left|\gamma^{\prime}\right|\right|_{\text {horizon }},
$$

where a prime, $\alpha$, and $\gamma$ are understood in the sense of the general metric (2.8) and $k_{\mathrm{B}}$ is the Boltzmann constant. The expression (6.17) is invariant with respect to radial coordinate reparametrization, as is necessary for any quantity having a direct physical meaning. Moreover, it can be shown to be invariant under conformal mappings if the conformal factor is smooth at the horizon.

Substituting $g_{00}$ and $g_{11}$ from $(\underline{6.10})$, one obtains:

$$
T=\frac{1}{2 \pi k_{\mathrm{B}}} \frac{1}{4 k}\left[\frac{4 k^{2}}{\left(2 k+p_{\mathrm{e}}\right)\left(2 k+p_{\mathrm{m}}\right)}\right]^{1 /\left(d_{2}+1\right)} .
$$

If $d_{2}=0$ and both charges are nonzero, this temperature tends to zero in the extreme limit $k \rightarrow 0$; if $d_{2}=1$ and both charges are nonzero, it tends to a finite limit, and in all other cases it tends to infinity. Remarkably, it is determined by the $p$-brane intersection dimension $d_{2}$ rather than the whole space-time dimension $D$.

\subsection{Examples}

Let us present some examples of configurations satisfying the orthogonality condition (6.5) with $\lambda=0$. This condition is then a Diophantus equation for $D, n$ and $d_{2}$. Some of its solutions are given in the following table, including also the values of the constants $B$ and $C$ defined in (6.14). 


\begin{tabular}{|c|c|c|c|c|c|c|}
\hline & $n$ & $d\left(I_{\mathrm{e}}\right)$ & $d\left(I_{\mathrm{m}}\right)$ & $d_{2}$ & $B$ & $C$ \\
\hline $\begin{array}{c}D=4 m+2 \\
=6,10,14,\end{array}$ & & & & & & \\
$18,22,26, \ldots$ & $2 m+1$ & $2 m$ & $2 m$ & $m-1$ & $1 / m$ & $1 / m$ \\
& & & & & & \\
& & & & & & \\
& 7 & 6 & 3 & 1 & $1 / 3$ & $2 / 3$ \\
$D=11$ & 7 & 6 & 12 & 3 & $1 / 3$ & $1 / 6$ \\
& 13 & 12 & 6 & 3 & $1 / 6$ & $1 / 3$ \\
\hline
\end{tabular}

Many of these configurations have been discussed in the literature on M-theory, probably the most well-known one is that of 2- (electric) and 5- (magnetic) branes intersecting along a string (1-brane) in $D=11$ supergravity.

\subsection{Special solution for a more general model}

Let us now cancel the orthogonality condition (6.5) (i.e. consider a more general set of input parameters) but suppose, as in Subsec. 4.2, $y_{\mathrm{e}}=y_{\mathrm{m}}$. As has been shown there, this implies $Q_{\mathrm{e}}^{2}=Q_{\mathrm{m}}^{2} \stackrel{\text { def }}{=} Q^{2}$.

The charges can be different only in the case $\lambda=d_{3}=d_{4}=0$, i.e. for a conformal field without dilatonic coupling, studied in [14, when the electric and magnetic $(n-2)$-branes coincide. In this and only in this case we have in (4.12)-(4.15) $Y_{1}=Y_{2}$. Then the charges $Q_{\mathrm{e}}$ and $Q_{\mathrm{m}}$ may be arbitrary but enter into the solution only in the combination $Q_{\mathrm{e}}^{2}+Q_{\mathrm{m}}^{2}$.

Let us study other cases. We are again work with $(6.1)-(6.3)$. With $y_{\mathrm{e}}=y_{\mathrm{m}} \stackrel{\text { def }}{=} y(u)$, Eq. (4.12) leads to

$$
d_{3} \beta_{3}-d_{4} \beta_{4}-2 \lambda \varphi=0
$$

Eqs. (4.14) are irrelevant since we are dealing with only two functions $y_{s}$. The equations of motion for $x^{A}$ now take the form

$$
x^{A^{\prime \prime}}=Q^{2} \mathrm{e}^{2 y}\left(Y_{\mathrm{e}}^{A}+Y_{\mathrm{m}}^{A}\right) .
$$

Their proper combination gives $y^{\prime \prime}=\left(1+d_{2}\right) Q^{2} \mathrm{e}^{2 y}$, whence

$$
\mathrm{e}^{-y}=\sqrt{\left(1+d_{2}\right) Q^{2}} s\left(h, u+u_{1}\right)
$$

where the function $s(.,$.$) is defined in (3.12) and h, u_{1}$ are ICs and, due to (6.4), $\sqrt{\left(1+d_{2}\right) Q^{2}} s\left(h, u_{1}\right)=1$. Other unknowns are easily determined using (6.20) and (6.4):

$$
\begin{aligned}
x^{A} & =\nu Y^{A} y+c^{A} ; \quad Y^{A}=Y_{\mathrm{e}}^{A}+Y_{\mathrm{m}}^{A}=(1,1,0,0,-1,0) ; \\
\sigma_{1} & =-\nu y+c_{0} u .
\end{aligned}
$$


Here, as in (6.7), $\nu=1 /\left(1+d_{2}\right)$, but it is now just a notation. The constants $c_{0}, h, c^{A}(A=$ $1, \ldots, 6)$ and $k$ (see $(3.11))$ are related by

$$
\begin{aligned}
& -c^{0}+\sum_{i=1}^{5} d_{i} c^{i}=0, \quad c^{1}+d_{2} c^{2}+d_{3} c^{3}-\lambda c^{6}=0, \quad c^{1}+d_{2} c^{2}+d_{4} c^{4}+\lambda c^{6}=0, \\
& 2 k^{2} \operatorname{sign} k=\frac{2 h^{2} \operatorname{sign} h}{1+d_{2}}\left(c^{0}\right)^{2}+\sum_{i=1}^{5} d_{i}\left(c^{i}\right)^{2}+\left(c^{6}\right)^{2} .
\end{aligned}
$$

This solution contains six independent ICs and, like that of Subsec. 6.1, directly generalizes many previous solutions, including those of Ref. [14. It is valid without restrictions upon the input parameters of the model. It actually repeats the solutions obtainable with a single charge, but with a more complicated space-time structure.

The only case when all extra-dimension scale factors remain finite as $u \rightarrow u_{\text {max }}$ is again that of a BH. It is specified by the following values of the ICs:

$$
k=h>0, \quad c^{3}=c^{4}=c^{6}=0, \quad c_{2}=-c_{5}=-\frac{k}{1+d_{2}}, \quad c_{0}=c^{1}=-\frac{d_{2} k}{1+d_{2}} .
$$

The event horizon occurs at $u=\infty$. After the same transformation (6.9) the metric takes the form

$$
\begin{aligned}
d s_{D}^{2}=-\frac{1-2 k / R}{(1+p / R)^{2 \nu}} d t^{2} & +(1+p / R)^{2 \nu}\left(\frac{d R^{2}}{1-2 k / R}+R^{2} d \Omega^{2}\right) \\
& +(1+p / R)^{-2 \nu} d s_{2}^{2}+d s_{3}^{2}+d s_{4}^{2}+(1+p / R)^{2 \nu} d s_{5}^{2}
\end{aligned}
$$

with the notation

$$
p=\sqrt{k^{2}+\left(1+d_{2}\right) Q^{2}}-k .
$$

The fields $\varphi$ and $F$ are determined by the relations

$$
\varphi \equiv 0, \quad F_{01 L_{3} \ldots L_{n}}=-\frac{Q}{R^{2}(1+p / R)}, \quad F_{23 L_{3} \ldots L_{n}}=Q \sin \theta .
$$

The mass and the Hawking temperature of such a BH, calculated as before, are given by the relations

$$
G_{N} M=k+p /\left(1+d_{2}\right), \quad T=\frac{1}{2 \pi k_{\mathrm{B}}} \frac{1}{4 k}\left(\frac{2 k}{2 k+p}\right)^{2 /\left(d_{2}+1\right)} .
$$

The well-known results for the Reissner-Nordström metric are recovered when $d_{2}=0$. In this case $T \rightarrow 0$ in the extreme limit $k \rightarrow 0$. For $d_{2}=1, T$ tends to a finite limit as $k \rightarrow 0$ and for $d_{2}>1$ it tends to infinity. As is the case with two different charges, $T$ does not depend on the space-time dimension $D$, but depends on the $p$-brane intersection dimension $d_{2}$.

\section{Concluding remarks}

We have seen that, in a model which may be called the electro-gravitational sector of M-theory, under certain restrictions fairly large classes of exact static, spherically symmetric solutions to 
the field equations can be obtained. Trying to be as close as possible to empirical practice, we restricted ourselves to a treatment of a single $F$-form and a 4-dimensional physical space-time.

The main results of possible physical significance are a non-hair-type theorem for quasiscalar components of an $F$-form and the behaviour of the BH temperature. The selection rules (3.9) for theories with multiple times are another point of interest.

We have left aside the problem of a physical 4-dimensional conformal frame, simply treating the 4-metric $g_{\mu \nu}=g_{M N} \quad(M, N=0, \ldots, 3)$ as a physical one. One reason is that the choice of a physical frame depends on the concrete form of the underlying theory, whereas this work discusses the weak field limit of a spectrum of theories, some of them are probably yet to be discovered. Some more details on this argument may be found in [21]. Furthermore, the question of the physical frame for effective (multi-)scalar-tensor theories (e.g. from multidimensional Einstein gravity) has been discussed in [17] (and further Refs. therein), concluding that, the question of the physical frame is not decidable with certainty on a purely classical level.

In any case, some important features of the solutions are independent of smooth conformal transformations of the frame. Thus, the $\mathrm{BH}$ nature of a solution and the Hawking temperature are insensitive to conformal factors which are smooth at the horizon. Furthermore, also the (highly anisotropic) singularities in non-BH solutions cannot be removed by smooth conformal transformations.

\section{Acknowledgement}

The authors are grateful for helpful discussions with V. Ivashchuk, V. Melnikov, and A. Zhuk. This work was financially supported by RFBR project grant N 95-02-05785-a, DFG grants 436 RUS 113/7, 436 RUS 113/236, KL 732/4-1, and Schm 911/6. It was partially completed at Astrophysikalisches Institut Potsdam and Institut für Mathematik, Universität Potsdam. K. B. wishes to express his gratitude to colleagues in Potsdam for their kind hospitality.

\section{References}

[1] C. Hull and P. Townsend, "Unity of Superstring Dualities", Nucl. Phys. B 438, 109 (1995), P. Horava and E. Witten, Nucl. Phys. B 460, 506 (1996), hep-th/9510209; hep-th/9603142.

[2] J.M. Schwarz, "Lectures on Superstring and M-theory Dualities", Preprint ICTP, hepth/9607201;

[3] K.S. Stelle, "Lectures on Supergravity p-branes ", hep-th/9701088.

[4] M.J. Duff, "M-theory (the Theory Formerly Known as Strings)", Preprint CTP-TAMU33/96, hep-th/9608117.

[5] J.P. Gauntlett, "Intersecting Branes", hep-th/970511.

[6] E. Cremmer, B. Julia and J. Scherk, Phys. Lett. B76, 409 (1978).

[7] A. Salam and E. Sezgin, eds., "Supergravities in Diverse Dimensions", reprints in 2 vols., World Scientific (1989). 
[8] H. Lü, C.N. Pope and K.W. Xu, "Liouville and Toda Solitons in M-theory", hepth/9604058.

[9] V.D. Ivashchuk and V.N. Melnikov, Gravitation 83 Cosmology 2, No 4 (8), 297 (1996); hep-th/9612089.

[10] V.D. Ivashchuk and V.N. Melnikov, Phys. Lett. B 384, 58 (1996).

[11] V. D. Ivashchuk, V. N. Melnikov, and M. Rainer. "Multidimensional $\sigma$-models with composite electric $p$-branes". gr-qc/9705005.

[12] I.Ya. Aref'eva, M.G. Ivanov and O.A. Rytchkov, "Properties of Intersecting p-branes in Various Dimensions", Preprint SMI-05-97, hep-th/9702077.

[13] I.Ya. Aref'eva, M.G. Ivanov and I.V. Volovich, hep-th/9702079.

[14] K.A. Bronnikov and J.C. Fabris, Gravitation 83 Cosmology 2, No 4 (8), 306 (1996)

[15] M. Rainer, Int. J. Mod. Phys. D 4, 397 (1995).

[16] M. Rainer, Gravitation \& Cosmology 1, 121 (1995).

[17] M. Rainer and A. Zhuk, Phys. Rev. D 54 (1996) 6186.

[18] M. Rainer, "Effective multi-scalar-tensor theories and $\sigma$-models from multidimensional gravity" Preprint Math-97/4, Univ. Potsdam (1997).

[19] K.A. Bronnikov, Acta Phys. Polon. B 4, 251 (1973).

[20] I. Bars and C. Kounnas, "Theories with Two Times", hep-th/9703060.

[21] K.A. Bronnikov, Gravitation \& Cosmology 1, 67 (1995).

[22] R. Wald, "General Relativity", Univ. of Chicago Press, Chicago, 1984. 